\title{
Genetic traceability of two local chicken populations, Bianca di Saluzzo and Bionda Piemontese, versus some current commercial lines
}

\author{
Stefano Sartore, Dominga Soglia, Sandra Maione, Paola Sacchi, Michele De Marco, \\ Achille Schiavone, Simone Sponza, Alessandra Dalmasso, Maria Teresa Bottero, \\ Daniele Pattono, Ivo Zoccarato, Laura Gasco, Alberto Brugiapaglia, Martina Tarantola, \\ Mario Giacobini, Luigi Bertolotti, Roberto Rasero \\ Scuola di Agraria e Medicina Veterinaria, Università di Torino, Italy
}

\begin{abstract}
The aims of this investigation were to analyse the genetic variation of two Piemonte chicken local breeds, Bionda Piemontese and Bianca di Saluzzo, and to set them against some commercial lines. A panel of 19 microsatellite markers was used. On the overall, the results of different analyses highlight the genetic uniqueness of the two breeds; therefore they should be considered genetic resources worthy of preservation. The panel of microsatellites used in this investigation turns out to be a consistent and reliable tool for traceability. In fact, these markers are able to distinguish the two local populations from the commercial lines and they are able to confirm the existence of two genetically different clusters within the Bionda Piemontese, namely the ecotypes standard and Cuneo. Mating policies implemented to avoid inbreeding and, if necessary, a marker assisted conservation scheme would be sufficient to solve the problem of inbreeding.
\end{abstract}

\footnotetext{
Correspondence: Stefano Sartore, Dipartimento di Scienze Veterinarie, Largo Paolo Braccini 2, 10095 Grugliasco (T0), Italy.

Tel.: +39.011.6709257 - Fax: +39.011.6709240.

E-mail: stefano.sartore@unito.it
}

Key words: Bianca di Saluzzo, Bionda Piemontese, chicken, local breeds, microsatellites, traceability.

Conference presentation: Meeting on Environmental Sustainability and Food Security, Potenza, Italy, 2014.

Acknowledgements: this work was supported by the grant of Università degli Studi di Torino and Compagnia di San Paolo for the project Innovative approaches for the control of poultry production chain (Alessandra Dalmasso, 2011).

Received for publication: 23 June 2014.

Revision received: 27 October 2014.

Accepted for publication: 8 November 2014.

CCopyright S. Sartore et al., 2014

Licensee PAGEPress, Italy

Italian Journal of Agronomy 2014; 9:605

doi:10.4081/ija.2014.605

This article is distributed under the terms of the Creative Commons Attribution Noncommercial License (by-nc 3.0) which permits any noncommercial use, distribution, and reproduction in any medium, provided the original author(s) and source are credited.

\section{Introduction}

The quality, rather than the quantity, of food of animal origin met recently the attention of the consumers, together with the growing perception that regional and traditional products could be healthier and tastier. This novel tendency is based on a reduced confidence towards food of animal origin, as a consequence of the outbreak of diseases like bovine spongiform encephalopathy, followed by avian influenza crisis (Ciampolini et al., 2000; Goffaux et al., 2005). Moreover, Opara and Mazaud (2001) reported a rising incidence of foodborne disease caused by microbial contamination of processed food, leading to additional mistrust in consumers.

Therefore an increasing need to check food processing aimed to prevent frauds and adulterations is arisen. The idea of traceability, intended to be a method able to identify animal or animal products through different steps of the food chain (McKean, 2001), became familiar to consumers, and the development of a reliable traceability system started to play a key role in this area of interest.

The EU always considered food safety a purpose of primary importance, first of all because it is the biggest producer of food and beverage of the world (European Commission, 2000). Following the regulation 178/2002 of the European Parliament, the traceability of food, fed, food-producing animals, and any other substance intended to be, or expected to be, incorporated into a food or feed shall be established at all stages of production, processing and distribution.

Then the concept of traceability turned out to be crucial both in safeguarding public and animal health and enhancing the value of typical products; consequently since 2005 it became mandatory for all member countries (Dalvit et al., 2005).

The efficiency of a traceability system is based on three pivotal points, i.e. the identification of the product, the collection of information on the product, and an integrated information management system (Nicoloso et al., 2013). The main biological source of information is DNA, which is inalterable, detectable, and permits the identification of individual, breed, and species, and whose analysis overtakes the limits of the traditional methods like ear tags (Cunningham and Meghen, 2001). In this regard, the use of microsatellite markers is one of the most common strategies, and it proved to be efficient in genetic characterisation and traceability of breeds belonging to different species like chicken (Rosenberg et al., 2001; Zanetti et al., 2010; Granewitze et al., 2014), sheep (Bramante et al., 2011; Lasagna et al., 2011), pig (Boitard et al., 2010; Wilkinson et al., 2011; Oh et al., 2014), cattle (Maudet et al., 2002; Moioli et al., 2004; Ciampolini et al., 2006; Orrù et al., 2006; Dalvit et al., 2008; Rodríguez-Ramírez et al., 2011; Rogberg-Muñoz et al., 2014), and fish (Yue et al., 2012).

Chicken meat is an important component of human nutrition. According to Zanon and Sabbioni (2001), the number of Italian local or autochthonous chicken breeds has dealt with a severe reduction in 
size, caused by replacement with cosmopolitan lines and highly productive crosses. The most dramatic effect of the intensive farming was the extinction of many local breeds (Gandini and Villa, 2003). Of the rural breeds present fifty years ago, $61 \%$ are currently extinct, $11 \%$ survive but are endangered, whereas only $7 \%$ have been involved in conservation programs.

In Piemonte region, two autochthonous breeds exist: Bianca di Saluzzo and Bionda Piemontese, both reared in the Asti and Cuneo provinces for meat production. In past decades, both breeds were subjected to a strong reduction in population size and were frequently replaced by fast-growing lines.

The aims of this investigation were to analyse the genetic variation of these breeds and to set them against some commercial lines. A panel of 23 microsatellite markers was used.

\section{Materials and methods}

\section{Sample collection}

A total of 540 blood samples belonging to the two Piemonte breeds and to four different commercial lines were collected: 213 from Bionda Piemontese (BP), 86 from Bianca di Saluzzo (BS), 61 from broiler chickens (BR), and 60 from each of three egg-laying commercial lines (CL), i.e. Hy-line, Isa Brown, and Eureka. Within the BP, the farmers usually distinguish two ecotypes based on different sampling areas and morphological traits, i.e. Bionda Piemontese standard (BPS) and Bionda Piemontese Cuneo (BPC); therefore we divided the BP into BPS (124 individuals) and BPC (89). The animals were chosen in different farms, namely 15 for BPC, 18 for BPS, and 6 for BS, in order to obtain a representative sample of each breed. DNA was extracted from blood specimens using the NucleoSpin QuickPure extraction kit (Macherey-Nagel, Dueren, Germany).

\section{Genotyping}

A set of 23 microsatellite loci was used, ADL0268, ADL0278, LEI0094, MCW0216, MCW0248, MCW0034, MCW0069, MCW0081, MCW0222, MCW0295, LEI0166, LEI0234, MCW0016, MCW0037, MCW0111, MCW0020, MCW0104, MCW0123, MCW0165, ADL0112, LEI0192, MCW0014, and MCW0183. These were part of the loci recommended by the Food and Agriculture Organisation (http://www.fao. org/docrep/ meeting/022/am652e.pdf Rome, 18-22 July 2011) and by the AVIANDIV project (2011) (http://aviandiv.tzv.fal.de/ primer_ table.html) and they are suitable for multiplex polymerase chain reaction (PCR) protocols. The markers were subjected to a multiplex PCR amplification in $10 \mathrm{~mL}$ reactions using the following final concentrations: 1X buffer Qiagen (Hilden, Germany), $0.4 \mathrm{mM}$ dNTPs, and 0.05 mM HotStartTaq Qiagen. The following thermo-cycling conditions were used: an initial denaturation step of $15 \mathrm{~min}$ at $95^{\circ} \mathrm{C}, 31$ cycles of $30 \mathrm{~s}$ at $95^{\circ} \mathrm{C}, 1 \mathrm{~min}$ at the annealing temperature specific to of each multiplex PCR, $1 \mathrm{~min}$ at $72^{\circ} \mathrm{C}$, and a final extension of $7 \mathrm{~min}$ at $72^{\circ} \mathrm{C}$. Analyses of fragments were performed using the automated DNA Genetic Analyzer ABI PRISM 310 (Applied Biosystems, Foster City, CA, USA) and the computer software GeneMapper 4.0 (Applied Biosystems). Allele calling was adjusted to Aviandiv project nomenclature including nine DNA reference samples. An error assay was performed by replicating the genotyping on a randomly chosen $10 \%$ of individual samples. The average error rate per locus was computed (Pompanon et al., 2005).

\section{Descriptive statistics for genetic diversity}

The main statistics were obtained using the software Fstat version 2.9.3.2 (Goudet, 1995) and GenAlEx version 3.2 (Peakall and Smouse, 2006, 2012). Observed number of alleles (A), allelic richness (R), number of private alleles (Ap), and observed (Ho) and unbiased expected $(\mathrm{He})$ heterozygosis were calculated by locus, overall loci, and by breed/line. Fis statistics per locus and breed/line and the significance of their non-zero values were performed (alleles were randomized among individuals within populations).

\section{Genetic differentiation and cluster analysis}

The Wright's F-statistics were calculated. Allele frequency differences across and between breeds/lines were tested with Fst statistics across all loci using the analysis of molecular variance. The deviation from the null hypothesis was tested with 999 permutations (GenAlEx 3.2). Average molecular coefficient of kinship (coancestry), fij, between $i$ and $j$ individuals within breed/line was also computed by bootstrapping (1000), equalising for sampling size (77), and weighted by the polymorphism information content of the marker loci (Molkin version 3.0, Gutiérrez et al., 2005).

The relationship between breeds/lines and individuals were represented using the FCA (factorial correspondence analysis) performed by the Génétix software (Lewis and Zaykin, 2001). A frequency-based population assignment test (Paetkau et al., 1995) was carried out and the leave-one-out procedure was used (GenAlEx 3.2).

The Bayesian methodology implemented by the Structure version 2.0 software was employed to determine the level of structure and substructure in the dataset (Pritchard et al., 2000). The assignment of individuals to breeds/lines assumed an ancestry model with admixture and correlated allele frequencies without prior information. Ten independent runs with 1,000,000 MCMC (Markov chain Monte Carlo) iterations and a burn-in period of 300,000 were carried out for $3 \leq K \leq 8$ number of clusters to estimate the most likely number of clusters (K) present in the dataset. The number of clusters was established by calculating $\Delta \mathrm{K}$ by the Structure Harvester software (Evanno et al., 2005; Earl and vonHoldt, 2012).

\section{Results}

Four microsatellites out of the 23 analysed were discarded because they scored a high error rate $(>0.02)$, so the genotyping data from 19 loci were finally used. The rate of missing genotypes was only $0.06 \%$.

All loci showed to be polymorphic and an overall number of 162 alleles was detected. The number of alleles per locus ranged from 26 (LEI0192) to 3 (MCW0248 and MCW0037).

The genetic variation within breed/line was quantified using the descriptive statistics of Table 1 . The average number of alleles was very similar to the average allelic richness; therefore differences in sample size across breeds/lines did not affect the genetic analyses.

Observed heterozygosis ranged from 0.64 in CL to 0.59 in BP, expected heterozygosis ranged from 0.67 in BPC to 0.55 in BR. The combined Fis values were significantly different from zero. Seven loci out of 19 in BPS, 8 in BPC, and 6 in BS contributed to heterozygosis deficiency. Removal of MCW0014, which showed the most difference $(\mathrm{P}<0.0001)$, did not reduce the value of significance. BR and CL showed heterozygosis excess. The average coefficient fij within breed/line was higher ( $>0.4$ ) in BR and CL than in the two local breeds (Table 2).

Variation among the breeds/lines, as estimated by the global Fst index, was highly significant ( $\mathrm{Fst}=0.109$, std. err. $0.017, \mathrm{P}<0.001$ ). All 
pairwise Fst values contributed with significant differences included the two ecotypes of $\mathrm{BP}(\mathrm{Fst}=0.035, \mathrm{P}<0.001)$ (Table 3$)$. The largest differences were obtained between the two local breeds and the other lines (Fst>0.100). Most private alleles were observed in the two local breeds, i.e. 13 in BP (4 in BPS and 9 in BPC) and 15 in BS (Table 4). Two alleles out of 4 showed a frequency above 0.01 in BPS, 6 out of 9 in BPC, and 10 out 15 in BS. The FCA plot of individual genotypes is presented in Figures 1 and 2. The distribution based on axis 1 (45\% of global genetic diversity) supported a clear difference between EL and the meat-producing chickens. Axis 2 (24\% of diversity) separated BP from BS and BR. The third factorial component (axis 3, 21\% of diversity) provided a further difference between BS and BR.

The pair wise assignment tests, as performed with the GenAlEx software, corroborated these results: BP and BS differed from BR and EL (data not shown). The assignment test gave $97 \%$ of correct individual assignments to the five clusters (Table 5). Four BPS individuals clustered together with BPC and 8 BPC with BPS. Two BP were assigned to EL and one BS to BR.

The Bayesian analysis performed by the Structure software showed that estimated posterior probability ( $\mathrm{Ln} \operatorname{Pr}(\mathrm{G} \mid \mathrm{K})$ ) reached plateau value at $\mathrm{K}=7(-24,672)$ but the modal value of the distribution of DK statistics was located at $\mathrm{K}=5(-24,959)$ (Figure 3$)$. The clustering pattern was as follow: BP split the two ecotypes BPS and BPC, BS, BR, and overall EL (Figure 4). Average membership of all breeds/lines except $\mathrm{BPC}$ was $>0.900$ in one of the five clusters (data not shown). In the present investigation, we were not able to distinguish the three lines of EL each other. Most incorrect individual assignments of BPS and BPC corresponded to the results obtained with the GenAlEx software, but no BP and BS were assigned to EL or BR.

\section{Discussion}

The panel of selected microsatellite markers revealed to be a good tool for diversity analyses of our local breeds. In a survey carried out on Spanish chicken breeds, Dávila et al. (2009) detected less than 4 alleles per locus. Hillel et al. (2003) assessed the genetic variation within and among 52 populations from a wide range of chicken types (local and commercial) and found an average of 3.52 alleles per locus. In Italian breeds, Bianchi et al. (2011) detected 3.73 alleles per locus in the Livorno, 3.50 in the Ancona, and 4.03 in Sasso.

The present investigation was carried out with the same panel of markers used by Hillel et al. (2003) and Bianchi et al. (2011) and reveals a similar or greater level of variation in the two Piemonte breeds, i.e. 5.89 alleles per locus in BPS, 6.37 in BPC, and 6.11 in BS, whereas less than 5 are present in broilers and layers.

In BP and BS the numbers of private alleles are not very high (13 and 15, respectively, $v s 17$ in Ancona and 26 in Livorno), nevertheless most of them show a frequency above 0.01 , so they may play a role in clustering success.

Dalvit et al. (2009) found a high and significant deficiency of heterozygosity in two Veneto local breeds, Ermellinata di Rovigo and

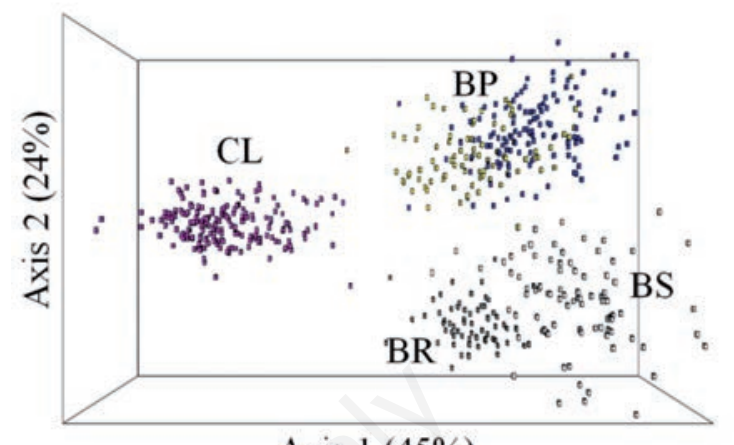

Axis $1(45 \%)$

Figure 1. Distribution of individuals based on the factorial correspondence analysis, axes 1 and 2 . BP, Bionda Piemontese; BS, Bianca di Saluzzo; BR, broiler chickens; CL, egg-laying commercial lines.

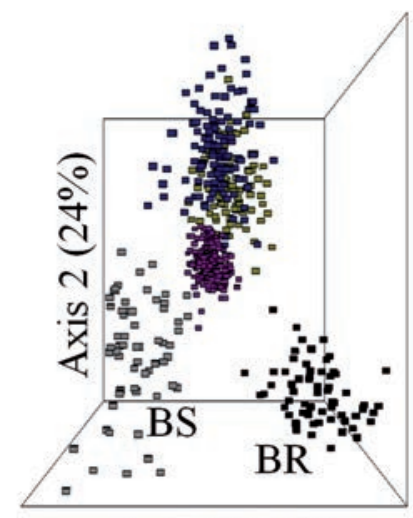

Axis $3(21 \%)$

Figure 2. Distribution of individuals based on the factorial correspondence analysis, axes 2 and 3. BS, Bianca di Saluzzo; BR, broiler chickens.

Table 1. Genetic diversity estimates over 19 marker loci for each breed/line included in this investigation.

\begin{tabular}{|c|c|c|c|c|c|c|c|c|}
\hline & $\mathbf{N}$ & A & $\mathbf{R}$ & Ap & Но & $\mathrm{He}$ & Fis & $\mathbf{P}$ \\
\hline BPS & 112 & $\begin{array}{l}5.89 \\
0.68\end{array}$ & 5.47 & 4 & $\begin{array}{l}0.59 \\
0.04\end{array}$ & $\begin{array}{l}0.66 \\
0.03\end{array}$ & +0.104 & $* * *$ \\
\hline BPC & 121 & $\begin{array}{l}6.37 \\
0.81\end{array}$ & 6.17 & 9 & $\begin{array}{l}0.59 \\
0.04\end{array}$ & $\begin{array}{l}0.67 \\
0.03\end{array}$ & +0.113 & $* * *$ \\
\hline BS & 116 & $\begin{array}{l}6.11 \\
0.67\end{array}$ & 5.86 & 15 & $\begin{array}{l}0.60 \\
0.04\end{array}$ & $\begin{array}{l}0.66 \\
0.03\end{array}$ & +0.089 & $* * *$ \\
\hline BR & 85 & $\begin{array}{l}4.47 \\
0.44\end{array}$ & 4.47 & 4 & $\begin{array}{l}0.61 \\
0.04\end{array}$ & $\begin{array}{l}0.55 \\
0.03\end{array}$ & -0.112 & $* * *$ \\
\hline CL & 88 & $\begin{array}{l}4.63 \\
0.47\end{array}$ & 4.37 & 3 & $\begin{array}{l}0.64 \\
0.07\end{array}$ & $\begin{array}{l}0.59 \\
0.04\end{array}$ & -0.087 & $* * *$ \\
\hline
\end{tabular}

$\mathrm{N}$, number of alleles; A, average observed number of alleles per locus; R, average allelic richness per locus; Ap, overall number of private alleles; Ho, observed heterozygosis; He, unbiased expected heterozygosis; BPS, Bionda Piemontese standard; BPC, Bionda Piemontese Cuneo; BS, Bianca di Saluzzo; BR, broiler chickens; CL, egg-laying commercial lines. ${ }^{* * *}<001$ (significance of non-zero values of Fis). 
Robusta Maculata. Over a 3-year period, the expected heterozygosity decreased from 0.54 to 0.42 and from 0.40 to 0.28 , respectively.

The average expected heterozygosity in the two Piemonte breeds is rather high and similar to the value of the Sasso breed ( 0.66 vs 0.60 ), a population which received a gene flow from other breeds (Bianchi $e t$ al., 2011). The average coefficients fij values within BP and BS were lower than those reported by Zanetti et al. (2011) for the other local Italian chicken breeds. Interestingly, compared to BP and BS, BR and EL show both heterozygosis excess and more co-ancestry. Foundation of commercial lines is based on different grandparent stocks from highly selected nuclei (Hillel et al., 2003; Tadano et al., 2007): if a limited number of stocks is used, these breeding practices may cause high observed heterozygosis in chickens sharing most alleles identical by descent. Actually, we have poor information about the phylogenetic relationships of $\mathrm{BP}$ and $\mathrm{BS}$, nevertheless the presence of private alleles and the differences emphasized by the cluster analysis shows that no recent introgression of allochtonous germplasm took place, in particular from commercial lines.

In fact, Sasso showed an excess of observed heterozygosis, just what happens to BR and CL in our investigation, which are obtained by specific crossings. On the opposite, significant deficiency of observed heterozygosis is present in BP and BS, as in other local breeds, which is usually a signature of inbreeding or sub-structuring in closed populations. This does not exclude that different populations could have concurred to foundation of BP and BS. All breeds received the genetic contribution of migration and admixture in the near or remote past. Afterwards, breeders concentrated on selection and genes migrant and native found their optimal proportions for a particular environment and production system (Nicholas, 2010).

This gene pool must be conserved. Mating policies and, if necessary, a marker assisted conservation scheme would be sufficient to limit the inbreeding. The existence of genetic differentiation between the two BP ecotypes is worth analysing in detail with further investigation. If such ecotypes have some attractive peculiarities, they would be preserved and exploited, otherwise their reproductive isolation just increases genetic subdivision, which will lead to additional homozygosity excess (Bianchi et al., 2011).

Table 2. Coefficient $f i j$ within breed/line.

\begin{tabular}{lll} 
& ffi & Standard error \\
BPS & 0.317 & 0.005 \\
BPC & 0.327 & 0.005 \\
\hline BS & 0.333 & 0.006 \\
BR & 0.435 & 0.008 \\
\hline CL & 0.415 & 0.006 \\
\hline
\end{tabular}

BPS, Bionda Piemontese standard; BPC, Bionda Piemontese Cuneo; BS, Bianca di Saluzzo; BR, broiler chickens; CL, egg-laying commercial lines.

Table 3. Fst (above the diagonal) and its significance (below the diagonal) as a measure of genetic differentiation between pairs of the breeds/lines.

\begin{tabular}{lccccc} 
& BPS & BPC & BS & BR & CL \\
BPS & - & 0.035 & 0.067 & 0.128 & 0.136 \\
BPC & $* * *$ & - & 0.072 & 0.110 & 0.103 \\
\hline BS & $* * *$ & $* * *$ & - & 0.124 & 0.131 \\
BR & $* * *$ & $* * *$ & $* * *$ & - & 0.151 \\
\hline
\end{tabular}

BPS, Bionda Piemontese standard; BPC, Bionda Piemontese Cuneo; BS, Bianca di Saluzzo; BR, broiler chickens; CL, egg-laying commercial lines. ${ }^{* * *} \mathrm{P}<0.001$ (significance of non-zero values).
The analysis of genetic diversity within and among breeds/lines was improved by the FCA and assignment test. The first three axes explained a high proportion of the total variation; therefore the separation of the two Piemonte breeds from each other and from the commercial lines is very reliable.

These results are confirmed by the Bayesian approach using the DK of Evanno et al. (2005), whose modal value is at the true K for most situations.

In the assignment tests, only few individuals moved away from the alleged cluster, two BP towards EL and one BS towards BR, but only with the Paetkau et al. (1995) test. The most important result is that no BR or EL were incorrectly assigned to BP or BS.

The clustering algorithm implemented by the Structure software has a great potential to correctly assign individuals of unknown origin (Rosenberg et al., 2001).

The panel of markers used in our investigation is a useful tool for traceability. This result is in agreement with the conclusions of Granevitze et al. (2014), who stated that microsatellite loci may perform even better than other marker types in the assignment tests applied to wild and domestic chicken populations.

In practice, the assignment tests show to be suitable tools to certify breed origin of meat and to detect adulterations of labelled products in chicken (Nakamura et al., 2006; Rikimaru and Takahashi, 2007), and also in pig (Oh et al., 2014), cattle (Ciampolini et al., 2006; Orrù et al.,
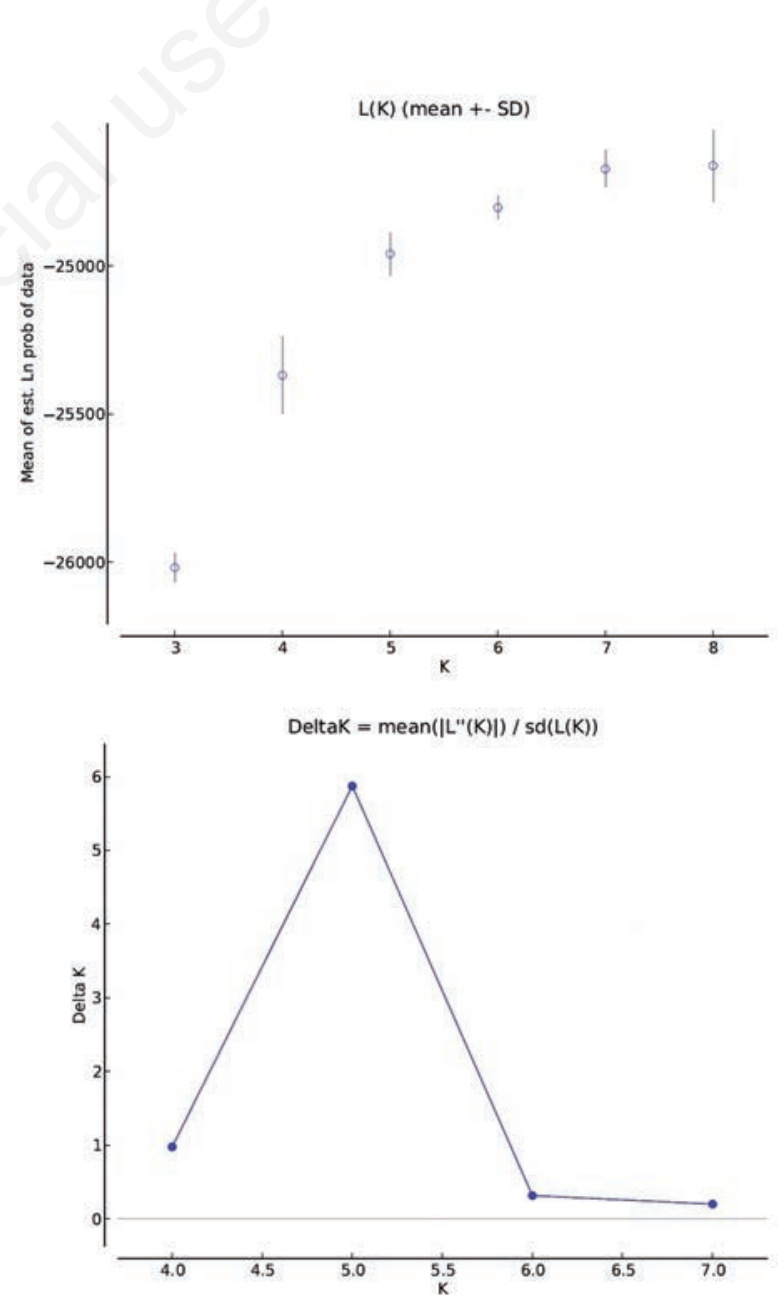

Figure 3. Graphic visualisation of estimated posterior probability of $\ln \operatorname{Pr}(G / K)=L(K)$ and delta $K$ distribution. 


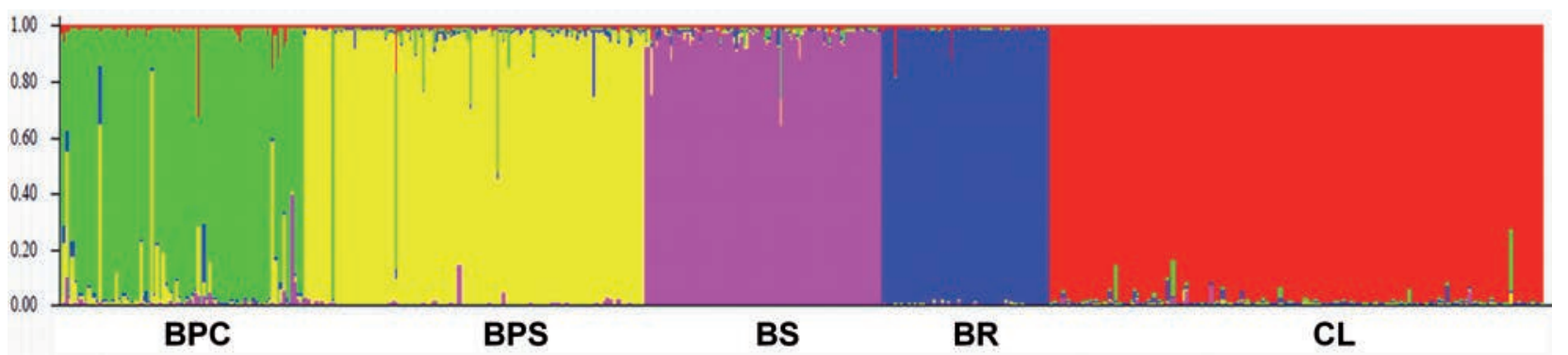

Figure 4. Bayesian cluster analysis at K=5. BPC, Bionda Piemontese Cuneo; BPS, Bionda Piemontese standard; BS, Bianca di Saluzzo; $\mathrm{BR}$, broiler chickens; CL, egg-laying commercial lines.

Table 4. Summary of private alleles in the two local breeds.

\begin{tabular}{cccc} 
& Locus & Allele (bp) & Frequency \\
BPS & MCW0104 & 204 & 0.028 \\
MCW0014 & 164 & 0.012 \\
ADL0112 & 122 & 0.008 \\
MCW0034 & 226 & 0.004 \\
LEI0192 & 245 & 0.028 \\
MCW0014 & 170 & 0.028 \\
LEI0192 & 249 & 0.022 \\
MCW0111 & 112 & 0.017 \\
LEI0192 & 283 & 0.017 \\
MCW0183 & 326 & 0.011 \\
LEI0094 & 241 & 0.006 \\
LEI0094 & 259 & 0.006 \\
LEI0192 & 257 & 0.006 \\
\hline MCW0034 & 212 & 0.064 \\
ADL0278 & 120 & 0.052 \\
MCW0081 & 122 & 0.052 \\
LEI0192 & 413 & 0.052 \\
MCW0016 & 206 & 0.041 \\
LEI0166 & 362 & 0.035 \\
LEI0094 & 275 & 0.029 \\
LEI0192 & 405 & 0.023 \\
MCW0014 & 182 & 0.017 \\
MCW0104 & 198 & 0.012 \\
ADL0278 & 112 & 0.006 \\
ADL0278 & 119 & 0.006 \\
MCW0216 & 149 & 0.006 \\
MCW0034 & 218 & 0.006 \\
LEI0166 & 358 & 0.006 \\
\hline BS & &
\end{tabular}

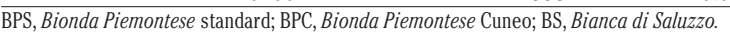

Table 5. Assignment test to correct (Self) or incorrect (Others) breed/line (Paetkau et al., 1995).

\begin{tabular}{lcc} 
& Self & Others \\
BPS & 119 & 5 \\
BPC & 80 & 9 \\
\hline BS & 85 & 1 \\
BR & 61 & 0 \\
\hline CL & 180 & 0 \\
Total & 525 & 15 \\
\hline Proportion & 0.97 & 0.03
\end{tabular}

BPS, Bionda Piemontese standard; BPC, Bionda Piemontese Cuneo; BS, Bianca di Saluzzo; BR, broiler chickens; CL, egg-laying commercial lines.
2006; Negrini et al., 2008; Rogberg-Muñoz et al., 2014), and fish (Yue et al., 2012).

\section{Conclusions}

On the overall, the results of different analyses highlight the genetic uniqueness of the two Piemonte breeds; therefore they should be considered genetic resources worthy of preservation. Secondly, the panel of 19 microsatellites used in this investigation turns out to be a consistent and reliable tool for traceability. In fact, these markers are able to distinguish the two local populations, BP and BS, from commercial lines and, moreover, they are able to confirm the existence of two genetically different clusters within the BP, namely BPS and BPC.

\section{References}

Aviandiv Project, 2011. Development of strategy and application of molecular tools to assess biodiversity in chicken genetic resources. Available from: http:/aviandiv.tzv.fal.de

Bianchi M, Ceccobelli S, Landi V, Di Lorenzo P, Lasagna E, Ciocchetti M, Sahin E, Mugnai C, Panella F, Sarti FM, 2011. A micro satellite-based survey on the genetic structure of two Italian local chicken breeds. It. J. Anim. Sci. 10:39.

Boitard S, Chevalet C, Mercat MJ, Meriaux JC, Sanchez A, Tibau J, Sancristobal M, 2010. Genetic variability, structure and assignment of Spanish and French pig populations based on a large scale sampling. Anim. Genet. 41:608-18.

Bramante A, Cecchi F, Ciani E, Castellana E, D'Andrea MS, Pilla F, Ciampolini R, 2011. The breed traceability of sheep meat by using molecular genetics methods: preliminary results. Ital. J. Food Safety 1:41-4.

Ciampolini R, Cetica V, Ciani E, Mazzanti E, Fosella X, Marroni F, Biagetti M, Sebastiani C, Papa P, Filippini G, Cianci D, Presciuttini S, 2006. Statistical analysis of individual assignment among four cattle breeds using fifteen STR loci. J. Anim. Sci. 84:11-9.

Ciampolini R, Leveziel H, Mozzanti E, Grohs C, Cianci D, 2000. Genomic identification of an individual or its tissue. Meat Sci. 54:35-40.

Cunningham EP, Meghen CM, 2001. Biological identification systems: genetic markers. Rev. Sci. Techn. 20:491-9.

Dalvit C, De Marchi M, Cassandro M, 2005. Genetic traceability of livestock products: a review. Meat Sci. 77:437-49.

Dalvit C, De Marchi M, Dal Zotto R, Gervaso M, Meuwissen T, Cassandro M, 2008. Breed assignment test in four Italian beef cattle breeds. 
Meat Sci. 80:389-95.

Dalvit C, Zanetti E, Cassandro M, 2009. Estimation of genetic diversity over time in an in-situ marker assisted conservation scheme of local chicken breeds. It. J. Anim. Sci. 8:63-5.

Dávila SG, Gil MG, Resino Talavan P, Campo JL, 2009. Evaluation of diversity between different Spanish chicken breeds, a tester line, and a White Leghorn population based on microsatellite markers. Poultry Sci. 88:2518-25.

Earl DA, vonHoldt BM, 2012. STRUCTURE HARVESTER: a website and program for visualizing STRUCTURE output and implementing the Evanno method. Conserv. Genet. Resour. 4:359-61.

European Commission, 2000. Council Regulation (EC) No. 1760/2000 of 17 July 2000 establishing a system for the identification and registration of bovine animals and regarding the labeling of beef and beef products and repealing Council Regulation (EC) No 820/97. Official Journal, L 204, 11/8/2000, pp 1-10.

Evanno G, Regnaut S, Goudet J, 2005. Detecting the number of clusters of individuals using the software STRUCTURE: a simulation study. Mol. Ecol. 14:2611-20.

Gandini GC, Villa E, 2003. Analysis of the cultural value of local livestock breeds: a methodology. J. Anim. Breed. Genet. 120:1-11.

Goffaux F, China B, Dams L, Clinquart A, Daube G, 2005. Development of a genetic traceability test in pig based on single nucleotide polymorphism detection. Forensic Sci. Int. 151:239-47.

Goudet J, 1995. FSTAT (version 1.2): a computer program to calculate Fstatistics. J. Hered. 86:485-6.

Granevitze Z, David L, Twito T, Weigend S, Feldman M, Hillel J, 2014. Phylogenetic resolution power of microsatellites and various singlenucleotide polymorphism types assessed in 10 divergent chicken populations. Anim. Genet. 45:87-95.

Gutiérrez JP, Royo LJ, Álvarez I, Goyache F, 2005. MolKin v2.0: a computer program for genetic analysis of populations using molecular coancestry information. J. Hered. 96:718-21.

Hillel J, Groenen MAM, Tixier-Boichard M, Korol AB, David L, Kirzhner VM, Burke T, Barre-Dirie A, Crooijmans RPMA, Elo K, Feldman MW, Freidlin PJ, Mäki-Tanila A, Oortwijn M, Thomsone P, Vignal A, Wimmers K, Weigend S, 2003. Biodiversity of 52 chicken populations assessed by microsatellite typing of DNA pools. Genet. Sel. Evol. 35:533-57.

Lasagna E, Bianchi M, Ceccobelli S, Landi V, Martinez Martinez A, Vega Pla JL, Panella F, Delgado Bermejo JV, Sarti FM, 2011. Genetic relationship and population structure in three Italian Merino-derived sheep breeds. Small Rumin. Res. 96:111-9.

Lewis PO, Zaykin D, 2001. Genetic data analysis: computer program for the analysis of allelic data. Version 1.0 (d16c). Free program available from: http//www.eeb.uconn.edu/people/plewis/software.php

Maudet C, Luikart G, Taberlet P, 2002. Genetic diversity and assignment tests among seven French cattle breeds based on microsatellite DNA analysis. J. Anim. Sci. 2002 80:942-50.

McKean JD, 2001. The importance of traceability for public health and consumer protection. Rev. Sci. Techn. 20:363-78.

Moioli B, Napolitano F, Catillo G, 2004. Genetic diversity between Piedmontese, Maremmana, and Podolica cattle breeds. J. Hered. 95:250-6.

Nakamura A, Kino K, Minezawa M, Noda K, Takahashi H, 2006. A method for discriminating a Japanese chicken, the Nagoya breed, using microsatellite markers. Poultry Sci. 85:2124-9.

Negrini R, Nicoloso L, Crepaldi P, Milanesi E, Marino R, Perini D, Pariset L, Dunner S, Leveziel H, Williams JL, Ajmone-Marsan P, 2008. Traceability of four European protected geographic indication (PGI) beef products using single nucleotide polymorphisms (SNP) and Bayesian statistics. Meat Sci. 80:1212-7.

Nicoloso L, Crepaldi P, Mazza R, Ajmone-Marsan P, Negrini R, 2013. Recent advance in DNA-based traceability and authentication of livestock meat PD0 and PGI products. Food Nutr. Agric. 5:9-18.

Nicholas FW, 2010. Introduction to veterinary genetics. $3^{\text {rd }}$ ed. WileyBlackwell, Chichester, UK.

Oh JD, Song KD, Seo JH, Kim DK, Kim SH, Seo KS, Lim HT, Lee JB, Park HC, Ryu YC, Kang MS, Cho S, Kim ES, Choe HS, Kong HS, Lee HK, 2014. Genetic traceability of black pig meats using microsatellite markers. Asian-Australas J. Anim. Sci. 27:926-31.

Opara L, Mazaud F, 2001. Food traceability from field to plate. Outlook Agric. 30:239-47.

Orrù L, Napolitano F, Catillo G, Moioli B, 2006. Meat molecular traceability: How to choose the best set of microsatellites? Meat Sci. 72:312-7.

Paetkau D, Calvert W, Stirling I, Strobeck C, 1995. Microsatellite analysis of population structure in Canadian polar bears. Mol. Ecol. 4:347-54.

Peakall R, Smouse PE, 2006. GenAlEx 6: genetic analysis in Excel. Population genetic software for teaching and research. Mol. Ecol. Notes. 6:288-95.

Peakall R, Smouse PE, 2012. GenAlEx 6.5: genetic analysis in Excel. Population genetic software for teaching and research - an update. Bioinformatics 28:2537 9.

Pompanon F, Bonin A, Bellemain E, Taberlet P, 2005. Genotyping errors: causes, consequences and solutions. Nat. Rev. Genet. 6:847-59.

Pritchard JK, Stephens M, Donnelly P, 2000. Inference of population structure using multilocus genotype data. Genetics 155:945-59.

Rikimaru K, Takahashi H, 2007. A method for discriminating a Japanese brand of chicken, the Hinai-jidori, using microsatellite markers. Poultry Sci. 86:1881-6.

Rodríguez-Ramírez R, Arana A, Alfonso L, González-Córdova AF, Torrescano G, Guerrero Legarreta I, Vallejo-Cordoba B, 2011. Molecular traceability of beef from synthetic Mexican bovine breeds. Genet. Mol. Res. 10:2358-65.

Rogberg-Muñoz A, Wei S, Ripoli MV, Guo BL, Carino MH, Castillo N, Villegas Castagnaso EE, Liron JP, Morales Durand HF, Melucci L, Villareal E, Peral-Garcia P, Wei YM, Giovambattista G,, 2014. Foreign meat identification by DNA breed assignment for the Chinese market. Meat Sci. 98:822-7.

Rosenberg NA, Burke T, Elo K, Guo BL, Carino MH, Castillo N, Villegas Castagnaso EE, Liron JP, Morales Durand HF, Melucci L, Villareal E, Peral-Garcia P, Wei YM, Giovambattista G,, 2001. Empirical evaluation of genetic clustering methods using multi locus genotypes from 20 chicken breeds. Genetics 159:699-713.

Tadano R, Nishibori M, Nagasaka N, Tsudzuki M, 2007. Assessing genetic diversity and population structure for commercial chicken based on forty micro satellite analyses. Poultry Sci. 86: 2301-8.

Wilkinson S, Haley C, Alderson L, Wiener P, 2011. An empirical assessment of individual-based population genetic statistical techniques: application to British pig breeds. Heredity. 106:261-9.

Yue GH, Xia JH, Liu P, Liu F, Sun F, Lin G, 2012. Tracing Asian seabass individuals to single fish farms using microsatellites. PLoS One 7:e52721.

Zanetti E, De Marchi M, Abbadi M, Cassandro M, 2011. Variation of genetic diversity over time in local Italian chicken breeds undergoing in situ conservation. Poultry Sci. 90:2195-201.

Zanetti E, De Marchi M, Dalvit C, Cassandro M, 2010. Genetic characterization of local italian breeds of chicken undergoing in situ conservation. Poultry Sci. 89:420-7.

Zanon A, Sabbioni A, 2001. Identificazione e salvaguardia genetic delle razze avicole italiane. Ann. Fac. Med. Vet. Parma. 21:117-34. 